'Departamento de Anestesiología y Reanimación. Facultad de Medicina. Universidad de Chile. Santiago, Chile.

${ }^{2}$ Servicio de Anestesiología. Hospital Clínico Universidad de Chile. Santiago, Chile. ${ }^{3}$ Unidad de Pacientes Críticos. Hospital Clínico Universidad de Chile. Santiago, Chile.

${ }^{4}$ Sistema de Atención Médica de Urgencia (SAMU), Región Metropolitana, Chile.

${ }^{5}$ Facultad de Medicina.

Universidad de Chile. Santiago, Chile.

${ }^{6}$ Universidad de los Andes. Santiago, Chile. aInterno de la carrera de Medicina.

${ }^{b}$ Enfermera universitaria 'Cirujano dentista.

${ }^{d}$ Doctor en Ciencias Médicas Universidad de Chile.

Trabajo no recibió financiamiento. Los autores declaran no tener conflictos de interés.

Recibido el 2 de agosto de 2018, aceptado el 17 de enero de 2019.

Correspondencia a: María Mercedes Aguirre C. Departamento de Anestesiología. Hospital Clínico Universidad de Chile,

Santos Dumont 999, Independencia, Santiago, Chile. mmaguirre@uchile.cl

\section{Registro de paro cardiorrespiratorio perioperatorio en un hospital universitario en el período 2006-2017}

\author{
MARÍA MERCEDES AGUIRRE C. ${ }^{1,2}$, SEBASTIÁN MAYANZ S.,3,4, \\ ALONSO BLANCH Z., ${ }^{1,2}$, HÉCTOR ARANIBAR L. ${ }^{5, a}$, \\ ALEJANDRO SALAZAR T.,, ,a , GIGIA ROIZEN G. ${ }^{5, a}$, \\ MARÍA GABRIELA ÁLVAREZ N. ${ }^{2, \mathrm{~b}}$, CARLOS IZQUIERDO A. ${ }^{6, \mathrm{c}}$, \\ ANTONELLO PENNA S., ${ }^{1,2, \mathrm{~d}}$
}

\section{Registry of perioperative cardiac arrests in a clinical hospital in the period 2006-2017}

Background: Perioperative cardiac arrest (PCA) is a rare but important event in the operating room. Aim: To describe PCA events at a Clinical Hospital in Santiago, Chile. Material and methods: Registry of PCA that occurred in the operating room (OR) and during procedures not carried out in the OR between September 2006 and November 2017. Precipitating events, type of anesthesia and results of resuscitation maneuvers were described. Results: Eighty events (five outside of the OR) during 170,431 surgical procedures were recorded, resulting in an incidence of 4.4 events per 10,000 interventions. Hypotension/hypoperfusion was the most frequently found preexisting condition (42.5\%). The main cause was the presence of preoperative complications (57.5\%). Nineteen cases (23.8\%) were attributable to anesthesia, with an incidence of 1.11 per 10,000 anesthetic procedures. Survival rate at hospital discharge was 52.5\%. The figure for PCA caused by anesthesia was $84.2 \%$. Conclusions: The incidence of PCA and its survival is similar to that reported abroad. In general, PCA has a better prognosis than other types of cardiac arrest, especially if it has an anesthetic cause.

(Rev Med Chile 2019; 147: 34-40)

Key words: Heart Arrest; Incidence; Intraoperative Complications; Operating Rooms; Registries.
$\mathrm{E}$ 1 riesgo de sufrir un paro cardiorrespiratorio perioperatorio (PCRP) durante un procedimiento quirúrgico o similar, lo tienen todos los pacientes y depende del estado preoperatorio del paciente y de factores propios del procedimiento y la técnica anestésica ${ }^{1}$.

La incidencia del PCRP ha disminuido, en concordancia con una caída en la mortalidad total perioperatoria de 10.603 por millón antes de la década 1970-79 a 1.176 por millón entre los años 1990 y $2000^{2}$. Lo mismo ha ocurrido con la mortalidad atribuible a la anestesia, disminuyendo desde 357 por millón antes de los años 70 a 34 por millón entre los años 1990 y $2000^{2}$. Esto se ha explicado por la aplicación de los estándares de monitorización intraoperatoria desde el año 1985 y por un mejor enfrentamiento del riesgo perioperatorio ${ }^{1,3}$.

La mortalidad del PCRP se conserva elevada (hasta 70\%) $)^{4}$, siendo mejor el pronóstico de sobrevida si el origen de este es atribuible a la anestesia ${ }^{5,6}$. Existe una gran variabilidad en las 
tasas publicadas de incidencia del PCRP, las cuales están entre los 2,3 y 163 casos de PCRP por cada 10.000 procedimientos $^{6-12}$.

Taylor y cols., en 1976, plantearon la necesidad de registrar los PCRP para conocer la incidencia, causas y pronóstico de estos, con el objetivo de establecer protocolos locales para disminuir la ocurrencia y mejorar los resultados de las medidas terapéuticas ${ }^{13}$. Buscando ese mismo objetivo, en el año 2006 se inició en el Hospital Clínico de la Universidad de Chile (HCUCH) el registro estandarizado y obligatorio de los PCRP.

\section{Material y Método}

Se presenta un estudio descriptivo de los registros de los eventos de PCRP ocurridos en la unidad de pabellones quirúrgicos y de procedimientos fuera de pabellón, entre septiembre de 2006 y noviembre de 2017. Se utilizó una pauta de recolección de datos al estilo Utstein modifica$\mathrm{da}^{14}$. Se obtuvo la aprobación del comité de ética institucional para la publicación de este registro.

Se definió como PCRP al evento en que un paciente requirió maniobras de compresiones torácicas o desfibrilación cardíaca, ocurrido desde el ingreso a la unidad del procedimiento hasta el momento del alta de la sala de recuperación de postanestesia. Se excluyeron los eventos en pacientes sometidos a cardiocirugía y los casos con orden de no reanimar. En caso de más de un evento por paciente, se registró solo el primero. Las maniobras de reanimación cardiopulmonar (RCP) se realizaron adhiriendo estrictamente a las recomendaciones vigentes de la International Liaison Committee on Resuscitation (ILCOR), publicadas por la American Heart Association en sus progresivas actualizaciones desde el $2005^{15-17}$.

Los datos fueron registrados por el anestesiólogo tratante del paciente o por el residente presente durante el evento, en conjunto con uno de los investigadores principales. Se registraron los siguientes datos: el tipo de anestesia administrada, las causas del PCRP, el grado de contribución de la anestesia, el primer ritmo cardiaco de PCRP reconocido, los resultados de las maniobras de RCP (vivo o muerto), la condición y el Cerebral Performance Category $(C P C)^{14}$, que refleja el estado neurológico funcional del paciente, al alta hospitalaria y a los 12 meses tras el evento. Además, se registró la información general del paciente, el
CPC previo al evento, las condiciones médicas o quirúrgicas preexistentes al momento de ocurrir el evento y si el procedimiento era de urgencia o electivo.

Se categorizaron las causas del PCRP en 4 grupos de acuerdo con lo propuesto por Irita y cols: 1) Complicaciones preoperatorias; 2) Eventos quirúrgicos intraprocedimiento; 3) Eventos médicos intraprocedimiento y 4) Atribuibles al manejo anestésico ${ }^{18}$. El grado de contribución de la anestesia al PCRP fue definido como atribuible o contribuyente por el médico anestesiólogo a cargo del paciente durante el evento junto a dos investigadores principales.

Los datos son expresados como medianas (rangos) y porcentajes, según el tipo de variable. Las bases de datos y los valores estadísticos se realizaron en Microsoft Office Excel 2010.

\section{Resultados}

Entre septiembre de 2006 y noviembre de 2017 se registraron 80 eventos, 75 casos (94\%) ocurrieron en la Unidad de Pabellón Quirúrgico y 5 , fuera de pabellón (Tabla 1).

En el período se realizaron 170.431 procedimientos en los pabellones quirúrgicos, con una incidencia de 4,4 PCRP por 10.000 procedimientos. La mediana de edad fue de 57 años (1-87), $54 \%$ de pacientes fueron mujeres. Al momento de ocurrir el evento el CPC era 1 en $78 \%$ de los pacientes (Tabla 2).

Las condiciones preexistentes más frecuentes fueron el estado de hipotensión/hipoperfusión en $42,5 \%$ (34 pacientes), insuficiencia hepática en $22,5 \%$ (18 pacientes), insuficiencia renal y enfermedad tumoral o hematológica en $21,25 \%$

\section{Tabla 1. Lugar de ocurrencia del paro cardiorrespiratorio perioperatorio}

\begin{tabular}{|lc|}
\hline Lugar & $\mathbf{n}=\mathbf{8 0}$ \\
\hline Área de pabellones quirúrgicos & $75(94 \%)$ \\
Dentro de la sala de operaciones & $71(89 \%)$ \\
Unidad de recuperación postanestésica & $4(5 \%)$ \\
Lugares remotos "fuera de pabellón" & $5(6 \%)$ \\
Sala de telecomandado (CPER) & $3(4 \%)$ \\
Sala de estudios electrofisiológicos & $1(1 \%)$ \\
Sala de angiografías & $1(1 \%)$ \\
\hline
\end{tabular}

CPER: Colangiopancreatografía endoscópica retrógrada. 


\section{Tabla 2. Cerebral performance category de los pacientes previo al paro cardiorrespiratorio perioperatorio}

\begin{tabular}{|c|c|}
\hline CPC previo al PCRP & n $\quad(\%)$ \\
\hline 1 & $62(77,5 \%)$ \\
\hline 2 & $6 \quad(7,5 \%)$ \\
\hline 3 & $4 \quad(5 \%)$ \\
\hline 4 & $1 \quad(1,25 \%)$ \\
\hline 5 & 0 \\
\hline No registrado & $7 \quad(8,75 \%)$ \\
\hline Total & 80 \\
\hline
\end{tabular}

CPC: Cerebral performance category; PCRP: Paro cardiorrespiratorio perioperatorio.

(17 pacientes), sepsis en $18,75 \%$ (15 pacientes) y alteraciones metabólicas en 15\% (12 pacientes), pudiendo sobreponerse algunas de ellas en ciertos casos. El 18,75\% (15 pacientes) no registró condiciones preexistentes (Tabla 3 ).

La distribución de los eventos fue similar entre procedimientos de urgencia $(48,75 \%)$ y electivos (51,25\%). El 81\% de los pacientes estaba bajo anestesia general (Tabla 4) y el primer ritmo identificado al momento de hacer el diagnóstico de paro cardiorrespiratorio fue un ritmo no desfibrilable (asistolía, actividad eléctrica sin pulso o bradicardia extrema) en 68 casos (85\%).

La principal causa definida fue la presencia de complicaciones preoperatorias $(57,5 \%)$. Los eventos quirúrgicos intraprocedimiento, médicos intraprocedimiento y atribuibles a la anestesia se registraron como causa en $23,8 \%$ de los eventos, cada uno (Tabla 5).

Entre las causas preoperatorias destacaron la hemorragia aguda e hipovolemia. La hemorragia aguda fue, además, la principal causa de los eventos de origen quirúrgico. En el caso de los eventos médicos intraoperatorios, la presencia de una arritmia nueva y la causa multifactorial de los pacientes sometidos a trasplante hepático fueron las más frecuentes.

Entre las causas atribuibles a la anestesia, se registraron 5 casos asociados a anestesia espinal, 4 casos secundarios a errores de medicación, 7 casos por hipoxemia ( 5 por manejo de la vía aérea y 2 por depresión respiratoria en el postoperatorio inmediato), un caso de desconexión inadvertida del circuito anestésico durante una anestesia ge-
Tabla 3. Condiciones preexistentes en el paciente al momento de presentar el paro cardiaco perioperatorio

\begin{tabular}{|c|c|}
\hline Condición preexistente & n (\%) \\
\hline Hipotensión/hipoperfusión & $34(42,5)$ \\
\hline Insuficiencia hepática & $18(22,5)$ \\
\hline $\begin{array}{l}\text { Enfermedad tumoral metastásica o } \\
\text { hematológica }\end{array}$ & $17(21,3)$ \\
\hline Insuficiencia renal & $17(21,3)$ \\
\hline Sepsis & $15(18,8)$ \\
\hline Alteración metabólica & $12(15)$ \\
\hline Alteración hidroelectrolítica & $11(13,8)$ \\
\hline Diabetes mellitus & $11(13,8)$ \\
\hline Arritmia & $9(11,3)$ \\
\hline Deterioro basal del SNC & $9(11,3)$ \\
\hline Insuficiencia respiratoria & $6 \quad(7,5)$ \\
\hline AVE & $5 \quad(6,3)$ \\
\hline Trauma (causa de la hospitalización actual) & $5 \quad(6,3)$ \\
\hline $\begin{array}{l}\text { Cardiopatía coronaria previa a hospitalización } \\
\text { actual }\end{array}$ & $4 \quad(5)$ \\
\hline $\begin{array}{l}\text { Insuficiencia cardiaca previa a hospitalización } \\
\text { actual }\end{array}$ & $4 \quad(5)$ \\
\hline Evento agudo del SNC no AVE & $3(3,8)$ \\
\hline Cardiopatía coronaria en hospitalización actual & $2 \quad(2,5)$ \\
\hline Insuficiencia cardiaca en hospitalización actual & $2(2,5)$ \\
\hline Neumonia & $1 \quad(1,3)$ \\
\hline Sin condiciones preexistentes & $15(18,8)$ \\
\hline
\end{tabular}

SNC: sistema nervioso central; AVE: accidente vascular encefálico. La sumatoria de todas las condiciones sobrepasa el número de eventos dado que hay pacientes que presentaron más de una condición en forma concomitante.

Tabla 4. Distribución de eventos por tipo de anestesia administrada

\begin{tabular}{|lr|}
\hline Tipo de anestesia & $\mathbf{n ~ ( 8 0 )}$ \\
\hline Anestesia general & $65(81,3)$ \\
\hline Anestesia espinal & $11(13,8)$ \\
\hline Anestesia general más bloqueo regional (plexo) & $1(1,3)$ \\
\hline Anestesia local & $1(1,3)$ \\
\hline Anestesia local más sedación & $1(1,3)$ \\
No especificado & $1(1,3)$ \\
Total & $180(100)$ \\
\hline
\end{tabular}


Tabla 5. Causas de paro cardiorrespiratorio perioperatorio

\begin{tabular}{|c|c|c|}
\hline Causas de PCRP & & n $(\%)$ \\
\hline $\begin{array}{l}\text { Complicaciones preoperatorias } \\
46(57,5 \%)\end{array}$ & $\begin{array}{l}\text { Hemorragia } \\
\text { Shock cardiogénico } \\
\text { Shock séptico } \\
\text { Trastorno hidroelectrolítico } \\
\text { Neumotórax }\end{array}$ & $\begin{array}{rr}16 & (20) \\
1 & (1,3) \\
9 & (11,3) \\
1 & (1,3) \\
1 & (1,3)\end{array}$ \\
\hline $\begin{array}{l}\text { Eventos quirúrgicos intraprocedimiento } \\
19(23,8 \%)\end{array}$ & $\begin{array}{l}\text { Hipovolemia } \\
\text { Hemorragia } \\
\text { Embolía aérea } \\
\text { Otro }\end{array}$ & $\begin{array}{cr}18 & (22,5) \\
10 & (12,5) \\
6 & (7,5) \\
3 & (3,8)\end{array}$ \\
\hline $\begin{array}{l}\text { Eventos médicos intraprocedimiento } \\
19(23,8 \%)\end{array}$ & $\begin{array}{l}\text { Arritmia } \\
\text { Trasplante } \\
\text { TEP } \\
\text { Síndrome coronario agudo }\end{array}$ & $\begin{array}{lr}9 & (11,3) \\
7 & (8,8) \\
1 & (1,3) \\
2 & (2,5)\end{array}$ \\
\hline $\begin{array}{l}\text { Atribuibles al manejo anestésico } \\
19(23,8 \%)\end{array}$ & $\begin{array}{l}\text { Errores de medicación } \\
\text { Hipoxia } \\
\text { Neumotórax a tensión } \\
\text { Asociado a anestesia espinal } \\
\text { Arritmia por CAP } \\
\text { Desconexión inadvertida de circuito anestésico }\end{array}$ & $\begin{array}{ll}4 & (5) \\
7 & (8,8) \\
1 & (1,3) \\
5 & (6,3) \\
1 & (1,3) \\
1 & (1,3)\end{array}$ \\
\hline Desconocido & & $3(3,8)$ \\
\hline
\end{tabular}

PCRP: Paro cardiorrespiratorio perioperatorio; TEP: Tromboembolismo pulmonar; CAP: Catéter de arteria pulmonar. La sumatoria de todas las causas sobrepasa el número de eventos, dado que hay pacientes en los cuales se consideró más de una causa desencadenante del evento.

neral, un caso de fibrilación ventricular durante la instalación de un catéter de arteria pulmonar y un caso de neumotórax a tensión durante la ventilación con máscara facial en la inducción anestésica.

La incidencia de PCRP atribuible a la anestesia resultó de 1,1 por 10.000 procedimientos. En 6 casos se consideró que la anestesia contribuyó al PCRP (0,35 por 10.000 procedimientos) (Tabla 6$)$.

Los resultados generales según la causa del PCRP se observan en la Tabla 7. La mortalidad

\section{Tabla 6. Contribución de la anestesia al paro cardiorrespiratorio perioperatorio}

\begin{tabular}{|lrc|}
\hline Grado de contribución de la anestesia & n & (\%) \\
\hline Atribuible a la anestesia & 19 & $(23,8)$ \\
\hline Anestesia contribuye parcialmente & 6 & $(7,5)$ \\
\hline La anestesia no contribuye & 52 & $(65)$ \\
\hline Desconocido & 3 & $(3,8)$ \\
Total & 80 & $(100)$ \\
\hline
\end{tabular}

Tabla 7. Resultados del PCRP

\begin{tabular}{|c|c|c|c|c|c|c|c|}
\hline Causa & $\begin{array}{l}\text { Condición } \\
\text { n (\%) }\end{array}$ & $\begin{array}{c}\text { Sobrevive } \\
\text { al PCRP }\end{array}$ & $\begin{array}{c}\text { Vivo al } \\
\text { alta }\end{array}$ & $\begin{array}{l}\text { Vivo a los } \\
12 \text { meses }\end{array}$ & $\begin{array}{c}\text { No cumple } \\
12 \text { meses de } \\
\text { seguimiento }\end{array}$ & $\begin{array}{c}\text { Alta } \\
\text { pendiente }\end{array}$ & Perdido \\
\hline \multicolumn{2}{|l|}{ Total } & $62(77,5)$ & $42(52,5)$ & $32(40)$ & $7(8,75)$ & $1(1,3)$ & $1(1,3)$ \\
\hline \multicolumn{2}{|c|}{ Condición preexistente } & $16(57,1)$ & $8(28,6)$ & $7(25)$ & $2(10,5)$ & $1(6,3)$ & $1(6,3)$ \\
\hline \multicolumn{2}{|c|}{ Evento quirúrgico intraprocedimiento } & $12(63,2)$ & $5(26,3)$ & $2(10,5)$ & $2(10,5)$ & - & - \\
\hline \multicolumn{2}{|c|}{ Evento médico intraprocedimiento } & $15(78,9)$ & $13(68,4)$ & $10(52,6)$ & $1(5,3)$ & - & - \\
\hline \multicolumn{2}{|c|}{ Causa anestésica } & $19(100)$ & $16(84,2)$ & $13(68,4)$ & $2(10,5)$ & - & - \\
\hline
\end{tabular}

Los porcentajes expresados en relación a las causas específicas representan la proporción en relación al total de su grupo específico, no del universo correspondientes a los 80 PCRP. 
al alta fue de 47,5\%. De los pacientes vivos al alta (38), el $C P C$ se mantuvo 1 o 2 en $65,8 \%$. En el seguimiento a 12 meses fallecieron 5 pacientes, todos durante los primeros 6 meses.

\section{Discusión}

Se estima una alta adhesión al sistema de registro de PCRP implementado en HCUCH desde el año 2006. Es posible una pérdida marginal de casos por subregistro no detectado por los métodos administrativos vigentes en la institución.

La incidencia de PCRP en la Unidad de Pabellón Quirúrgico observada en el período estudiado (11 años y 2 meses) en el HCUCH, es de 4,4 por 10.000 procedimientos. Esta cifra se encuentra en el límite inferior de lo publicado internacionalmente, que establece cifras entre 2,3 y 34,6 por cada 10.000 pacientes sometidos a anestesia ${ }^{5,7,8,10-12,19}$. No hay datos publicados a nivel nacional.

$\mathrm{Al}$ no existir un registro unificado en $\mathrm{HCUCH}$ de los procedimientos que requirieron anestesiólogo de apoyo fuera de la Unidad de Pabellón Quirúrgico, no es posible establecer la tasa de PCRP "fuera de pabellón".

La mortalidad total al alta de nuestra serie es de 4,7 por 10.000 procedimientos, lo cual está dentro del rango reportado $(1,41 \text { a } 28,2 \text { por } 10.000)^{24}$. La sobrevida al alta en nuestra serie es de $52,5 \%$, concordante y levemente más alta que lo publicado ${ }^{20}$.

Respecto a las causas de PCRP, destaca que la mayoría corresponde a una causa no anestésica $(77,7 \%)$ y los eventos se distribuyen en forma homogénea entre las diferentes causas. La incidencia de causa anestésica en nuestra serie es de 1,11 por 10.000 anestesias y la de la contribución de la anestesia es de 0,35 por 10.000 anestesias. Esta cifra es similar a lo publicado, donde se reportan incidencias desde 0,5 a 2,10 por 10.000 anestesias ${ }^{5}$. Nuestra tasa de contribución de la anestesia resultó baja en comparación con lo publicado por Ellis y cols, probablemente por diferencias de criterios que implica esta definición.

Relacionando causa y pronóstico, 47,3\% de los pacientes que sobreviven al evento presentan una causa anestésica. La mortalidad atribuible a la anestesia fue de 0,17 por 10.000 anestesias, lo que está en el rango de lo publicado $(0,12$ a 1,4 por 10.000 anestesias $)^{5,19}$. Por el contrario, en los sobrevivientes al alta y al año de seguimiento, la causa de eventos quirúrgicos intraprocedimientos se encuentra solo en $13,1 \%$ y $6,9 \%$, respectivamente.

Además, de la elevada sobrevida al alta del PCRP anestésico (84,2 \%), destaca que $80 \%$ de los pacientes se recuperan con $C P C 1$ a 2, lo que traduce preservación de función neurológica ${ }^{14}$. En nuestra serie, la causa secundaria a condiciones preexistentes y la causa atribuible a eventos quirúrgicos intraprocedimiento, presentan la menor sobrevida al alta y a los 12 meses $(28,6 \%$ y $25 \%$; $26,3 \%$ y $10,5 \%$, respectivamente), mientras que la causa atribuible a eventos médicos intraprocedimiento, se relaciona con mayor sobrevida al alta y a los 12 meses (68,4\% y 52,6\%, respectivamente).

Estas cifras coinciden con la tendencia publicada, la cual muestra que el PCRP relacionado a la anestesia es de mejor pronóstico que aquel atribuido a una causa quirúrgica o a condiciones previas del paciente. En la serie de Nunes y cols, las cifras de sobrevida son de $50 \%, 28,6 \%$ y $31 \%$, respectivamente ${ }^{20}$.

Con relación al detalle de las causas anestésicas del PCRP, en esta serie se confirma lo ya publicado. La hipoxemia o problemas de manejo de la vía aérea y el error de medicación ocupan los primeros lugares ${ }^{5,9,10,21,22}$. El PCRP asociado a anestesia espinal es secundario a hipoperfusión por reflejo de Bezold Jarish o caída del retorno venoso con buena y rápida respuesta a las maniobras de $\mathrm{RCP}^{11}$.

Es importante señalar que 78\% de los PCRP atribuibles a la anestesia se consideraron prevenibles. Se cumple lo planteado por Biboulet y cols, quienes sostenían que cerca de $90 \%$ de los PCRP anestésicos están determinados por la presencia del error humano en alguna parte del proceso, ya sea una inadecuada evaluación preoperatoria del paciente, errada estimación de riesgo, errónea planificación de manejo de vía aérea, error en la dosificación de anestésicos, entre otros ${ }^{22}$.

Propio del paro cardiorrespiratorio intrahospitalario, el primer ritmo diagnosticado en nuestro universo fue un ritmo no desfibrilable mayoritariamente $(85 \%)^{23}$. Sin embargo, la mortalidad general fue de $52 \%$. Esto subraya lo expuesto por Ramachandran y cols., estos ritmos ominosos en otros escenarios deben tratarse agresivamente en el perioperatorio dadas sus características particulares, siempre buscando la causa, la cual muchas veces resulta fácilmente identificable y efectivamente tratable ${ }^{24}$. 
En nuestra serie, $80 \%$ de los PCRP se asociaron a anestesia general, consistente con lo descrito y conociendo que los pacientes más graves o con mayor riesgo, se intervienen bajo esta técnica anestésica $^{10,25}$.

Finalmente, reafirmamos el objetivo último de este trabajo de larga data, el cual es buscar el mejor resultado para nuestros pacientes. Visualizamos así la necesidad de mantener la educación y capacitación periódica trasversal en la institución, así como de adoptar y reforzar toda medida de seguridad y competencia técnica que permita mantener o mejorar los datos presentados.

\section{Referencias}

1. Villarreal R, Alvarado M, Torres RO. Principales causas anestésicas de paro cardiaco. Rev Sanid Mil Mex 1991; 45: 83-5.

2. Bainbridge D, Martin J, Arango M, Cheng D. Perioperative and anaesthetic-related mortality in developed and developing countries: a systematic review and meta-analysis. The Lancet 2012; 380 (9): 1075-81.

3. Irita K, Tsuzaki K, Sanuki M, Sawa T, Nakatsuka H, Makita K, et al. Recent changes in the incidence of life-threatening events in the operating room: JSA surveys between 2001 and 2005. Masui 2007; 56 (12): 1433-46.

4. Fachini M, Ximenes R, Fabio G, Da Luz V, Sá L, Castro $\mathrm{M}$, et al. Parada cardíaca perioperatória: uma análise evolutiva da incidência de parada cardíaca intraoperatória em centros terciários no Brasil, Rev Bras Anestesiol 2016; 66 (2): 176-82.

5. Ellis S, Newland M, Simonson J, Reed Peters K, Romberger D, Mercer D, et al. Anesthesia-related Cardiac Arrest. Anesthesiology 2014; 120: 829-38.

6. Hernández YI. Paro cardiaco transoperatorio. Rev Colomb Anestesiol 2002; 30 (2) Disponible en: http://www. redalyc.org/articulo.oa?id=195118154006 [Consultado el 26 de julio de 2018].

7. Nunnally ME, O’Connor MF, Kordylewski H, Westlake $\mathrm{B}$, Dutton RP. The incidence and risk factors for perioperative cardiac arrest observed in the national anesthesia clinical outcomes registry. Anesth Analg 2015; 120 (2): 364-70.

8. Newland MC, Ellis SJ, Lydiatt CA, Peters KR, Tinker JH, Romberger DJ, et al. Anesthetic Related Cardiac Arrest and its Mortality. A report covering 72959 Anesthetics over 10 years from US Teaching Hospital. Anesthesiology 2002; 97: 108-15.

9. Sprung J1, Warner ME, Contreras MG, Schroeder DR,
Beighley CM, Wilson GA, et al. Predictors of survival following cardiac arrest in patients undergoing noncardiac surgery: a study of 518,294 patients at a tertiary referral center. Anesthesiology 2003; 99: 259-69.

10. Braz L, Módolo N, Do Nascimento P, Bruschi B, Castiglia Y, Ganem E, et al. Perioperative cardiac arrest: a study of 53718 anaesthetics over 9 yr from a Brazilian teaching hospital, Br J Anaesth 2006; 96: 596-75.

11. Aguirre M. Paro Cardiorrespiratorio Perioperatorio. Rev Chil Anest 2012; 41 (1): 13-7.

12. Ahmed A, Ali M, Khan EA, Khan MU. An audit of perioperative cardiac arrests in a Southeast Asian university teaching hospital over 15 years. Anaesth Intensive Care 2008; 36 (5): 710-6.

13. Taylor G, Larson CP, Prestwich R. Unexpected cardiac arrest during anesthesia and surgery. An environmental study. JAMA 1976; 236 (24): 2758-60.

14. Jacobs I, Nadkarni, Bahr J, Berg RA, Billi JE, Bossaert L, et al. Cardiac arrest and cardiopulmonary resuscitation outcome reports: update and simplification of the Utstein templates for resuscitation registries: a statement for healthcare professionals from a task force of the International Liaison Committee on Resuscitation (American Heart Association, European Resuscitation Council, Australian Resuscitation Council, New Zealand Resuscitation Council, Heart and Stroke Foundation of Canada, InterAmerican Heart Foundation, Resuscitation Councils of Southern Africa). Circulation 2004; 110 (21): 3385-97.

15. 2005 International consensus on cardiopulmonary resuscitation $(\mathrm{CPR})$ and emergency cardiopulmonary resuscitation (CPR) and emergency cardiovascular care (ECC) science with treatment recommendations. Circulation 2005; 112 (22) supplement.

16. Travers AH, Rea TD, Bobrow BJ, Edelson DP, Berg RA, Sayre MR, et al. CPR overview: 2010 American Heart Association Guidelines for Cardiopulmonary Resuscitation and Emergency Cardiovascular Care. Circulation 2010; 122: S676-84.

17. Neumar RW, Shuster M, Callaway CW, Gent LM, Atkins DL, Bhanji F, et al. 2015 American Heart Association Guidelines Update for Cardiopulmonary Resuscitation and Emergency Cardiovascular Care. Circulation 2015; 132 (18 Suppl 2): S315-67.

18. Irita K, Kawashima Y, Iwao Y, Seo N, Tsuzaki K, Morita $\mathrm{K}$, et al. [Annual mortality and morbidity in operating rooms during 2002 and summary of morbidity and mortality between 1999 and 2002 in Japan: a brief review]. Masui 2004; 53 (3): 320-35.

19. Pignaton W, Braz J, Kusano P, Módolo M, de Carvalho L, Braz M, et al. Perioperative and Anesthesia-Related 
Mortality An 8-Year Observational Survey From a Tertiary Teaching Hospital. Medicine 2016; 95 (2): e2208.

20. Nunes JC, Braz JRC, Oliveira TS, de Carvalho LR, Castiglia YMM, Braz LG. Intraoperative and Anesthesia-Related Cardiac Arrest and Its Mortality in Older Patients: A 15-Year Survey in a Tertiary Teaching Hospital. Landoni G, ed. PLoS ONE 2014; 9 (8): e104041.

21. Moitra V, Einav S, Thies KC, Nunnally M, Gabrielli A, Maccioli G, et al. Cardiac Arrest in the Operating Room: Resuscitation and Management for the Anesthesiologist: Part 1. Anesth Analg 2018; 126 (3): 876-88.

22. Biboulet P, Aubas P, Dubourdieu J, Rubenovitch J, Capdevila X, d'Athis F. Fatal and non fatal cardiac arrests related to anesthesia. Can J Anaesth; 2001; 48 (4): 326-32.
23. Peberdy MA, Kaye W, Ornato JP, Larkin GL, Nadkarni $\mathrm{V}$, Mancini ME, et al. Cardiopulmonary resuscitation of adults in the hospital: a report of 14720 cardiac arrest from the National Registry of Cardiopulmonary Resuscitation. Resuscitation 2003; 58: 297-308.

24. Ramachandran SK, Mhyre J, Kheterpal S, Christensen RE, Tallman K, Morris M, et al. Predictors of survival from perioperative cardiopulmonary arrests: a retrospective analysis of 2,524 events from the Get With The Guidelines-Resuscitation registry. Anesthesiology 2013: 119 (6): 1322-39.

25. Braz LG, Braz DG, Da Cruz DS, Fernandes LA, Módolo NSP, Braz JRC. Mortality in Anesthesia: A Systematic Review. Clinics (Sao Paulo) 2009; 64 (10): 999-1006. 